\section{Case Reports in Dermatology}

Case Rep Dermatol 2017;9:86-90

DOI: $10.1159 / 000477456$

Published onlıne: July 13, 2017

(C) 2017 The Author(s)

Published by S. Karger AG, Basel

www.karger.com/cde

This article is licensed under the Creative Commons Attribution-NonCommercial 4.0 International License (CC BY-NC) (http://www.karger.com/Services/OpenAccessLicense).

Usage and distribution for commercial purposes requires written permission.

\title{
Sweet's Syndrome Arising in a Scar
}

\author{
Casey M. Chern ${ }^{a} \quad$ Michael Royer $^{b} \quad$ Winston Turnage $^{c}$ \\ ${ }^{a}$ WRNMMC Dermatology, Bethesda, MD, USA; ${ }^{b}$ WRNMMC Pathology, Bethesda, MD, USA; \\ 'Tripler Army Medical Center, Honolulu, HI, USA
}

\section{Keywords}

Sweet's syndrome $\cdot$ Neutrophilic dermatosis $\cdot$ Karyorrhexis $\cdot$ Koebnerization

\begin{abstract}
Acute febrile neutrophilic dermatosis (Sweet's syndrome) is an uncommon inflammatory cutaneous disorder. It presents with lesions which are tender, erythematous, edematous papules and under histologic examination show dense neutrophilic infiltration of the dermis. These lesions are often accompanied by leukocytosis and fever. This paper reports a unique case of acute febrile neutrophilic dermatosis presenting as a Koebner response to a linear scar.

(C) 2017 The Author(s)

Published by S. Karger AG, Basel
\end{abstract}

\section{Introduction}

Acute febrile neutrophilic dermatosis (Sweet's syndrome) is an uncommon inflammatory cutaneous disorder. It presents with lesions which are tender, erythematous, edematous papules and plaques typically involving the face, neck, upper trunk, and extremities. This is typically accompanied by fever and leukocytosis with more than $75 \%$ of patients having systemic symptoms [1]. Sweet's syndrome primarily affects adults, with a 3:1 female dominance. Infections are acute and self-limited and often follow an upper respiratory tract infection [2]. Koebnerization after trauma, such as this case, may uncommonly occur. 


\section{Case Report}

A 68-year-old Thai woman was admitted to the hospital with acute onset of tender nodules developing in a surgical scar on her left knee, as well as on her face, back, arms, and thighs. She was otherwise asymptomatic without a history of fevers, chills, joint pain, or gastrointestinal symptoms.

Her past history was significant for a left total knee replacement performed 6 months prior to presentation. The surgery was complicated by a methicillin-resistant Staphylococcus aureus infection requiring surgical washout 3 months after the initial procedure. Eight days prior to presentation, the patient was started on trimethoprim-sulbactam for an unrelated axillary abscess. Her initial lab values were remarkable for leukocytosis, with a white blood cell count of 11.9 and thrombocytopenia with a platelet count of 22,000.

Her physical exam revealed multiple, indurated, red-to-violaceous, juicy papules and plaques most pronounced on the left knee in the area of a linear, vertically oriented surgical scar. Pinpoint 1-mm petechiae were observed diffusely over the chest (Fig. 1, Fig. 2).

Histologically, two punch biopsies were performed to fresh appearing lesions on the left forearm for hematoxylin and eosin (H\&E) staining and tissue culture. The H\&E-stained sections were notable for a neutrophilic dermatosis with leukocytoclasis and mild papillary dermal edema. Some extravasated erythrocytes were present, but overt findings of small vessel vasculitis, such as perivascular fibrinous exudate or destruction of vascular walls, were not seen. Histochemical stains for organisms (PAS for fungus, Brown-Hopps for bacteria, and Ziehl-Neelsen for acid fast organisms) were negative. Biopsy for tissue culture was negative for deep fungal or atypical mycobacterial infection (Fig. 3, Fig. 4).

No evidence of associated malignancy was revealed in the patient course consisting of an age-appropriate malignancy workup. The most likely etiology for Sweet's syndrome in this case was thought to be the addition of trimethoprim-sulfamethoxazole in the weeks prior to presentation. The appearance of cutaneous lesions of Sweet's syndrome in a surgical scar is unique. A review did not reveal similar reported cases in the literature. There are reported cases of Sweet's syndrome exhibiting koebnerization (isomorphic phenomenon) at sites of trauma or sun exposure, and we propose that this may be an example of the same.

Our patient was deemed to be a poor candidate for systemic corticosteroids given for her underlying thrombocytopenia and recent infection. She was treated conservatively with topical triamcinolone ointment for symptomatic relief. Trimethoprim-sulfamethoxazole therapy was discontinued.

At a 2-week follow-up visit the patient's cutaneous lesions were nearly resolved with minimal intervention. Associated leukocytosis and thrombocytopenia had also improved at that time.

\section{Discussion}

Acute febrile neutrophilic dermatosis (Sweet's syndrome) is the prototypic neutrophilic dermatosis, and has been associated with a host of drug exposures, infections, malignancies (especially acute myelogenous leukemia), inflammatory and autoimmune diseases, and pregnancy [3]. The abrupt onset of tender, nonpruritic, juicy, red papules, and plaques is typical. Fever and constitutional symptoms are also common. The cutaneous lesions are selflimited and generally resolve without intervention within weeks of presentation. The erup- 
tion is typically very responsive to systemic steroids; however, recurrences occur in up to $30 \%$ of patients [4].

Proposed diagnostic criteria for Sweet's syndrome include two major criteria of abrupt onset of typical cutaneous lesions and histopathology consistent with Sweet's (i.e., diffuse neutrophilic infiltration in the dermis with karyorrhexis and massive papillary edema) [5]. In addition, four proposed minor criteria include the presence of fever and constitutional signs and symptoms, leukocytosis, response to systemic steroids, or preceding respiratory infection, drug exposure, pregnancy, known associated malignancy or inflammatory disorder [6]. Both major and two minor criteria are necessary for a diagnosis of Sweet's syndrome.

\section{Statement of Ethics}

The patient has given informed consent.

\section{Disclosure Statement}

The authors have no conflicts of interest to disclose.

\section{References}

1 Cohen PR: Sweet's syndrome - a comprehensive review of an acute febrile neutrophilic dermatosis. Orphanet J Rare Dis 2007;2:34.

2 Kemmett D, Hunter JAA: Sweet's syndrome: a clinicopathologic review of 29 cases. J Am Acad Dermatol 1990;23:503-507.

3 Azfar RS, Cohn J, Schaffer A, Kim EJ: Trimethoprim sulfamethoxazole-induced sweet syndrome. Arch Dermatol 2009;145:215-216.

4 Wallach D, Vignon-Pennamen MD: From acute febrile neutrophilic dermatosis to neutrophilic disease: forty years of clinical research. J Am Acad Dermatol 2006;55:1066-1071.

5 Su WPD, Liu HONH: Diagnostic criteria for Sweet's syndrome. Cutis 1986;37:167-174.

-6 Von den Driesch P: Sweet's syndrome: acute febrile neutrophilic dermatosis. J Am Acad Dermatol 1994;31:535-556. 


\section{Case Reports in Dermatology}

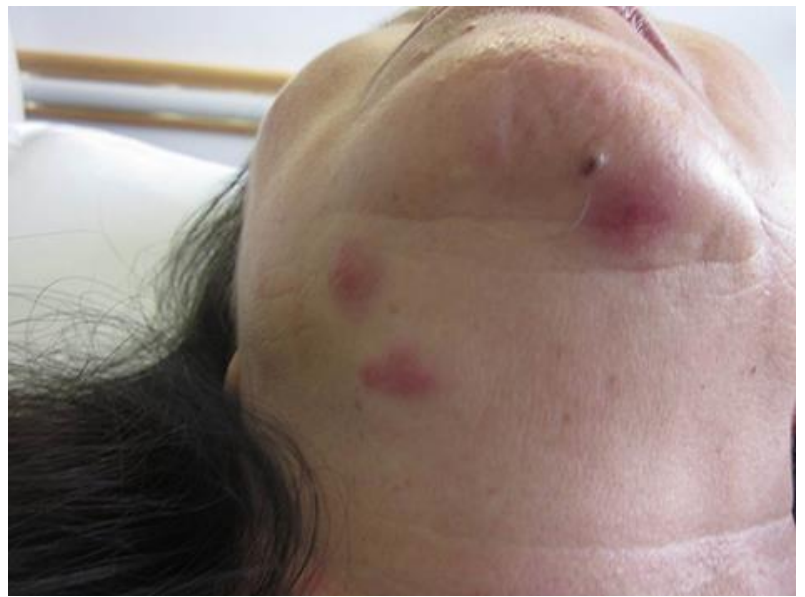

Fig. 1. Red indurated plaques on central face, chin, and neck of patient.

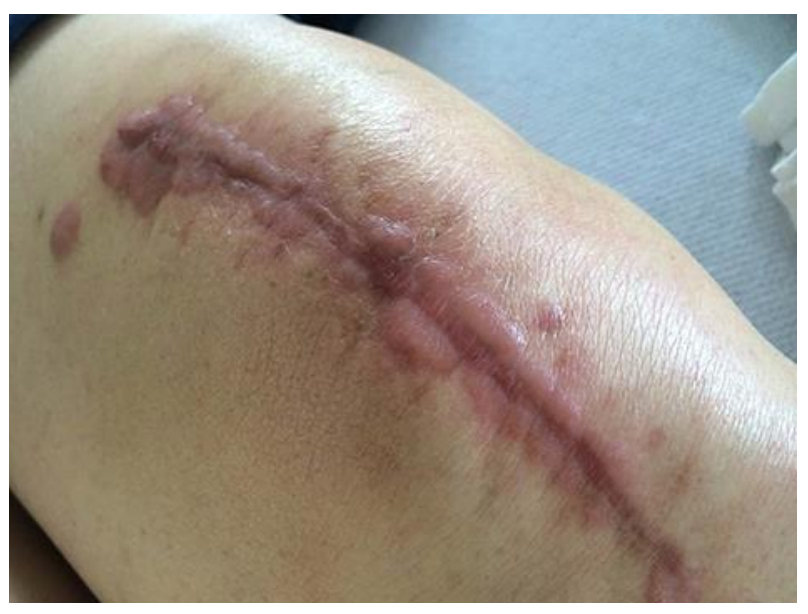

Fig. 2. Erythematous-to-violaceous indurated papules and plaques most pronounced on the left knee in the area of a linear, vertically oriented surgical scar. 


\section{Case Reports in

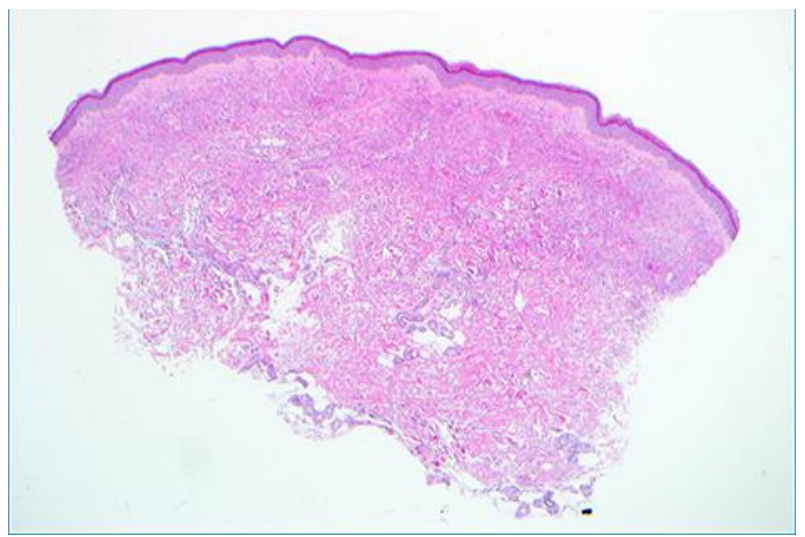

Fig. 3. Neutrophilic dermatosis with leukocytoclasis and mild papillary dermal edema. H\&E. Original magnification $\times 20$.

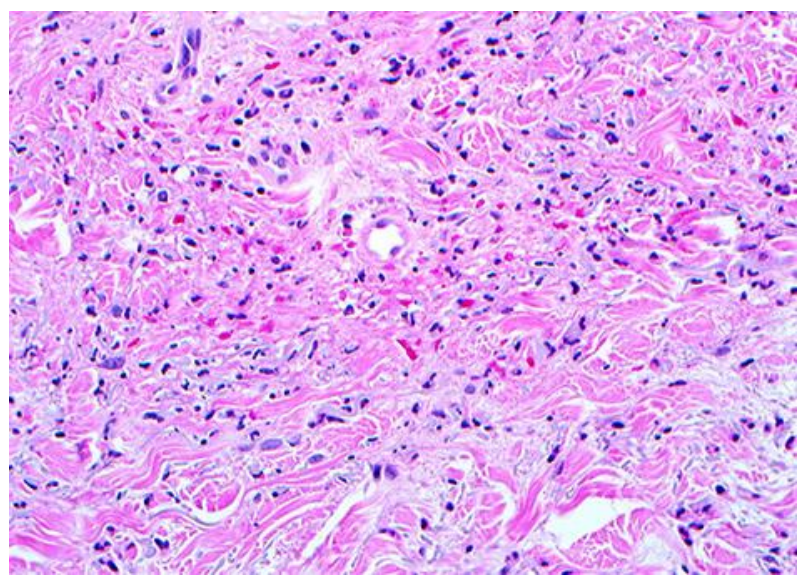

Fig. 4. Neutrophilic dermatosis with leukocytoclasis and mild papillary dermal edema. H\&E. Original magnification $\times 200$. 